\title{
Dephasing in the quasi-two-dimensional exciton-biexciton system
}

\author{
Langbein, Wolfgang Werner; Hvam, Jørn Märcher
}

Published in:

Physical Review B Condensed Matter

Link to article, DOI:

10.1103/PhysRevB.61.1692

Publication date:

2000

Document Version

Publisher's PDF, also known as Version of record

Link back to DTU Orbit

Citation (APA):

Langbein, W. W., \& Hvam, J. M. (2000). Dephasing in the quasi-two-dimensional exciton-biexciton system. Physical Review B Condensed Matter, 61(3), 1692-1695. https://doi.org/10.1103/PhysRevB.61.1692

\section{General rights}

Copyright and moral rights for the publications made accessible in the public portal are retained by the authors and/or other copyright owners and it is a condition of accessing publications that users recognise and abide by the legal requirements associated with these rights.

- Users may download and print one copy of any publication from the public portal for the purpose of private study or research.

- You may not further distribute the material or use it for any profit-making activity or commercial gain

- You may freely distribute the URL identifying the publication in the public portal

If you believe that this document breaches copyright please contact us providing details, and we will remove access to the work immediately and investigate your claim 


\title{
Dephasing in the quasi-two-dimensional exciton-biexciton system
}

\author{
W. Langbein \\ Lehrstuhl für Experimentelle Physik EIIb, Universität Dortmund, Otto-Hahn Strasse 4, D-44221 Dortmund, Germany \\ J. M. Hvam \\ Research Center COM, Technical University of Denmark, Building 349, DK-2800 Lyngby, Denmark
}

(Received 4 August 1999)

\begin{abstract}
The polarization decay in the exciton-biexciton system of a homogeneously broadened single quantum well is studied by transient four-wave mixing. All three decay rates in the exciton-biexciton three-level system are deduced. The relation between the rates unravels correlations between scattering processes of excitons and biexcitons. Density and temperature dependences show that the involved processes are mainly radiative decay and phonon scattering. The radiative decay rate of the biexcitons is found to be comparable to the one of the excitons, and the involved spontaneous photon emissions from excitons and biexcitons are mutually uncorrelated. In contrast, the biexciton phonon scattering is twice as fast and correlated to exciton-phonon scattering, indicating the interaction with similar phonon modes.
\end{abstract}

The enhancement of the Coulomb interaction between electrons and holes in semiconductor nanostructures leads to pronounced excitonic effects. This gives a strong influence on the optical nonlinearity not only from excitons but also from biexcitons. The binding energy of the biexcitons has been investigated in great detail, ${ }^{1-3}$ while only little is known about the scattering processes of biexcitons ${ }^{4-6}$ compared to that of excitons. ${ }^{7}$ In four-wave mixing (FWM) spectroscopy, commonly used to measure scattering processes, the disorder present in nanostructures strongly modifies the FWM response, ${ }^{8}$ especially the biexcitonic signal,,${ }^{9,10}$ and the analysis of the biexciton scattering is intricate. ${ }^{11}$ In a homogeneously broadened system instead, the dephasing rates of exciton and biexciton to ground-state transitions and also of the exciton-biexciton transition can be determined. This gives the opportunity to analyze not only the biexciton scattering processes but also their correlation with the exciton scattering processes, providing insight into the microscopic dynamics of the involved scattering events. A prominent example of a strong correlation of such scattering events is a slow dephasing of intraband coherences created by interband transitions of fast dephasing, like heavy-hole-light-hole beats $^{12,13}$ or Bloch oscillations. ${ }^{14}$

In bulk $\mathrm{CuCl}$, the biexciton acoustic-phonon scattering and the radiative decay was determined by two-photon absorption and time-resolved photoluminescence. ${ }^{6,15}$ The measured radiative lifetime of about $50 \mathrm{ps}$ is in agreement with a bipolariton model suggested by Ivanov et al. ${ }^{16}$ The acousticphonon scattering was assigned to phonon absorption. In CdSe the induced exciton-biexciton transition was investigated by FWM, ${ }^{5,17}$ showing a dephasing time of $20 \mathrm{ps}$ and an acoustic-phonon scattering with an activation energy given by the biexciton binding energy. In quasi-two-dimensional GaAs structures, the giant oscillator strength model gives comparable radiative decay rates for excitons and biexcitons, ${ }^{18,19}$ while the bipolariton model $^{20,21}$ predicts a fast decay of biexcitons into interface polaritons. An initial fast transient in the secondary emission of GaAs multiple quantum wells (QW's) (Ref. 19) was attributed to this fast decay. However, a direct measurement of the biexciton scattering in quasi-two-dimensional systems is still missing.

Here we study a homogeneously broadened quasi-twodimensional exciton-biexciton system in a $\mathrm{GaAs} / \mathrm{Al}_{0.3} \mathrm{Ga}_{0.7} \mathrm{As}$ single quantum well of $25 \mathrm{~nm}$ thickness grown by molecular-beam epitaxy. The sample is placed in a helium cryostat at a temperature between 5 and $80 \mathrm{~K}$. The absorption spectrum determined from photoluminescence (PL) at $20 \mathrm{~K}$ assuming a thermal distribution [Fig. 1(a)] shows a ground-state heavy-hole exciton peak ( $1 s$ hh) with a linewidth of $\gamma_{X}=75 \mu \mathrm{eV}$. All linewidths $\gamma$ given in this paper are half-width at half maximum and are related to the polarization decay time $T_{2}$ by $\gamma=\hbar / T_{2}$. We determine the linewidths in the exciton-biexciton system by spectrallyresolved, time-integrated FWM in reflection geometry, using excitation pulses in the directions $\mathbf{k}_{1}$ and $\mathbf{k}_{2}$ with $\tau_{12}$ temporal separation. The pulses from a mode-locked Ti:sapphire laser are chirp compensated and spectrally shaped to a duration of about $500 \mathrm{fs}$. The pulse spectra were adjusted to overlap only with the $1 s$ hh exciton and exciton-biexciton transition. The emitted FWM signal in the $2 \mathbf{k}_{2}-\mathbf{k}_{1}$ direction is selected spatially by pinholes and detected spectrally resolved by a combination of a spectrometer and an optical multichannel analyzer with a resolution of $0.08 \mathrm{meV}$. The excited exciton densities were $1-5 \times 10^{9} / \mathrm{cm}^{2}$.

Polarization selection rules in FWM can be used to discriminate transitions from the ground state of the crystal $|0\rangle$ to one of the optically active exciton states $X$, or transitions from $X$ to a biexciton state (bound $X X$ or unbound $X X^{*}$ ). The selection rules are derived from a five-level optical Bloch equation model ${ }^{22,23}$ indicated in Fig. 1(d). Due to the dominant homogeneous broadening, the FWM signal is a free polarization decay, which was confirmed by real-time resolved experiments. Using colinearly polarized $\mathbf{k}_{1}$ and $\mathbf{k}_{2}$ pulses $(\uparrow \uparrow)$, the FWM signal at the energy $E_{X}$ of the $|0\rangle-X$ transition $(X)$ is due to excitation-induced dephasing 24,25 (EID), local fields ${ }^{26}$ (LF), and phase-space filling (PSF). This gives rise to a complicated delay-time dependence of the 

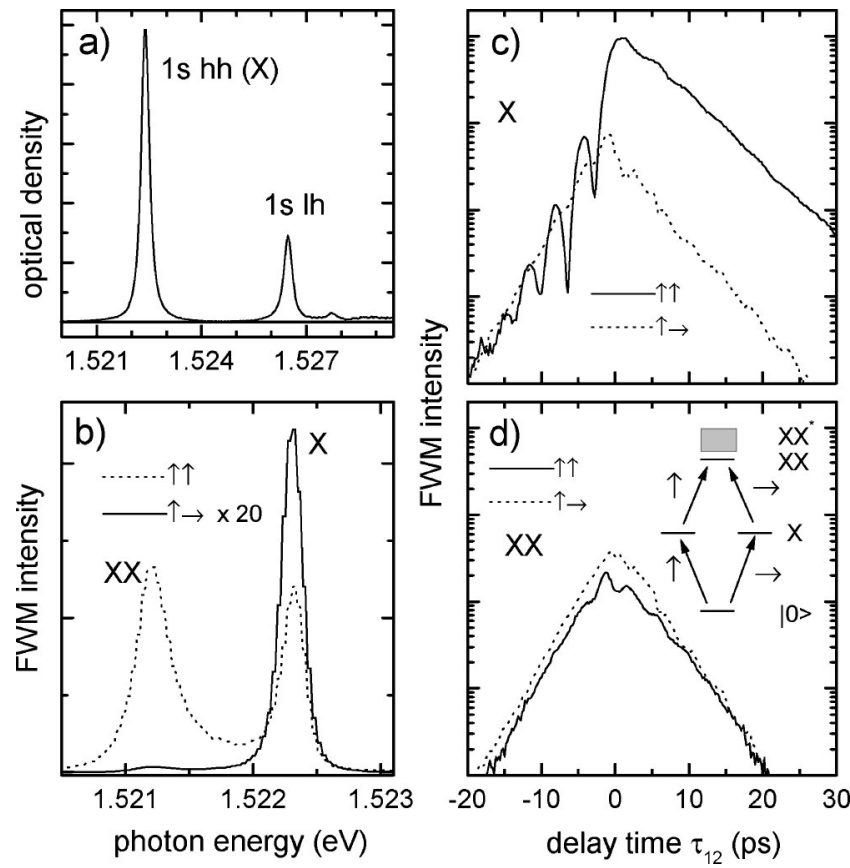

FIG. 1. Optical properties of the 25-nm GaAs SQW. (a) Optical density deduced from the photoluminescence at $20 \mathrm{~K}$. (b) Fourwave mixing spectra at $\tau_{12}=1 \mathrm{ps}$ and different polarization configurations, as indicated. The $(\uparrow \rightarrow)$ data are multiplied by 20 . The exciton density was about $10 \% / \mathrm{cm}^{2}$. (c),(d) Four-wave mixing traces detected at $(\mathrm{c})$ the exciton $(X)$ and $(\mathrm{d})$ the exciton-biexciton $(X X)$ transition, for different polarization configurations as indicated. A sketch of the five-level system for a linearly polarized exciton basis is shown in the inset of (d).

signal [see Fig. 1(c)], which cannot be easily analyzed. Instead, the signal resonant to the $X-X X$ transition energy $E_{X X}(X X)$ has only one origin, namely, PSF for $\tau_{12}>0$ and two-photon coherence (TPC) for $\tau_{12}<0$. For $(\uparrow \uparrow)$ polarization, a strong EID-induced FWM signal resonant at $E_{X}$ is disturbing the signal resonant at $E_{X X}$. It can be suppressed using cross linearly polarized $\mathbf{k}_{1}$ and $\mathbf{k}_{2}$ pulses $(\uparrow \rightarrow)$. For this polarization configuration, only the LF and the $X-X X^{*}$ contributions resonant at $E_{X}$ are left, which are not significantly disturbing the signal resonant at $E_{X X}$. Consequently, the signal decay at $E_{X X}$ is given for $\tau_{12}<0$ by the linewidth $\gamma_{X X g}$ of the $|0\rangle-X X$ transition due to TPC 27 , while for $\tau_{12}$ $>0$ it is given by the linewidth $\gamma_{X}$ of the $|0\rangle-X$ transition. The experimental FWM traces [Fig. 2(a)] show the expected exponential decay in both delay directions. For large delays $\left|\tau_{12}\right| \gtrsim 10 \mathrm{ps}$, the decay gets slightly faster, possibly a sign of residual inhomogeneous broadening ${ }^{9,28,10}$ or memory effects in the exciton-exciton scattering as discussed in Ref. 29. The FWM spectra [Fig. 2(b)] consist of the two contributions resonant to $E_{X X}$ and $E_{X X^{*}}$, that are shifting with temperature due to the band-gap shift of GaAs. These spectra can be fitted by the coherent superposition of two Lorentzian emission lines with amplitudes $A_{X X, X X^{*}}$, transition energies $E_{X X, X X^{*}}$, and linewidths $\gamma_{X X, X X^{*}}$ :

$$
I(\hbar \omega) \propto\left|\frac{A_{X X}}{\hbar \omega-E_{X X}+i \gamma_{X X}}+\frac{A_{X X^{*}}}{\hbar \omega-E_{X X^{*}}+i \gamma_{X X^{*}}}\right|^{2} .
$$
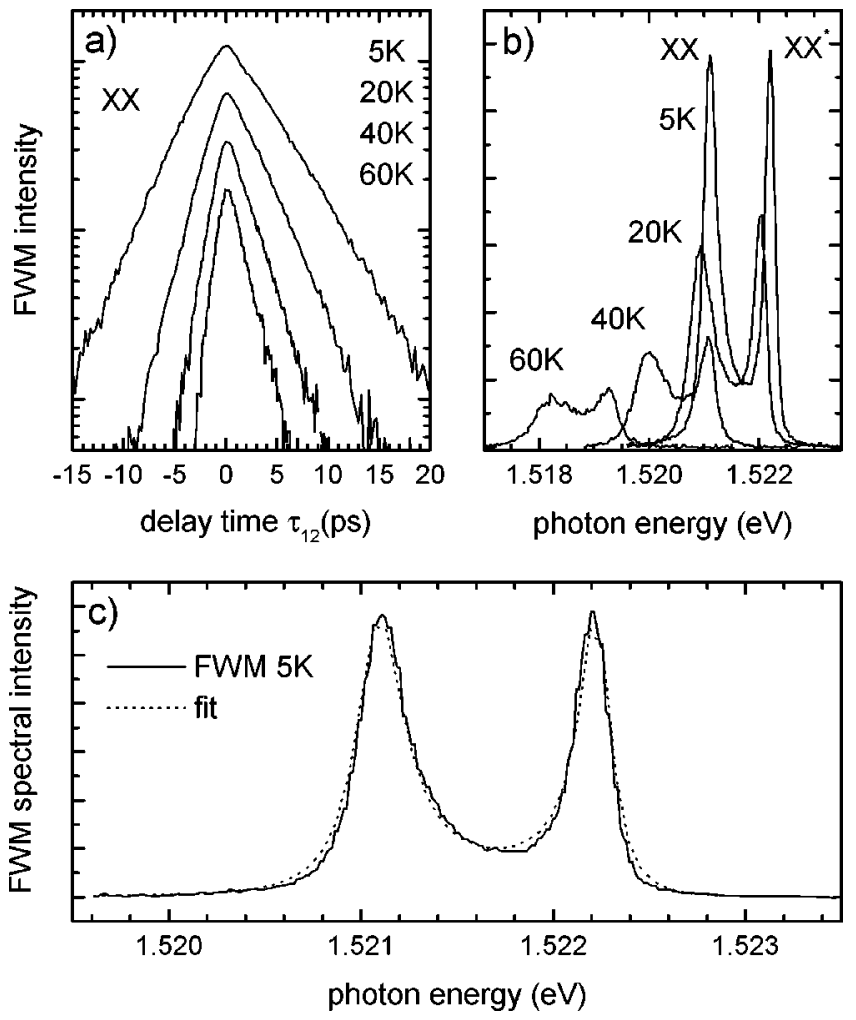

FIG. 2. (a),(b) Temperature dependency of the four-wave mixing signal for $(\uparrow \rightarrow)$ configuration. (a) Delay-time traces detected at the exciton-biexciton transition. (b) Spectra for $\tau_{12}=1 \mathrm{ps}$. (c) Fourwave mixing spectrum at $\tau_{12}=1 \mathrm{ps}$, and $5 \mathrm{~K}$ lattice temperature, together with a fit using Eq. (1).

This fit is used to extract the linewidth of the excitonbiexciton transition $\gamma_{X X}$, and an example for $T=5 \mathrm{~K}$ is shown in Fig. 2(c). The biexciton binding energy taken as the difference between $E_{X X}$ and $E_{X}$ is $1.1 \mathrm{meV}$. The energy $E_{X X^{*}}$ and the linewidth $\gamma_{X X^{*}}$ is found to be similar to those of the exciton $E_{X}$ and $\gamma_{X}$. This is expected since the localization of the excitons due to inhomogeneous broadening is negligible in the investigated sample, and the exciton-exciton continuum edge is at twice the exciton energy. ${ }^{28}$ Due to the continuum of scattering states, few-level models are not well suited to describe the nonlinear response in this spectral region, ${ }^{30}$ and more advanced theoretical approaches are needed. ${ }^{30-32}$ However, in the polarization configuration used, the level model is applicable to extract the linewidths of the transitions between the bound states in the system.

The linewidths $\gamma_{X}, \gamma_{X X g}$, and $\gamma_{X X}$ taken from the FWM traces and FWM spectra are extrapolated to zero exciton density and displayed in Fig. 3. Additionally, data from Lorentzian line-shape fits to the excitonic PL are shown, which are in agreement with the FWM data. The exciton linewidth $\gamma_{X}$ can be fitted with the expected dependence for exciton-phonon scattering: ${ }^{33}$

$$
\gamma=\gamma_{0}+a T+\frac{b}{\exp \left(E_{\mathrm{LO}} / k_{\mathrm{B}} T\right)-1}
$$

with the acoustic- and optical-phonon coefficient $a$ and $b$, and the zero-density and zero-temperature extrapolated line- 

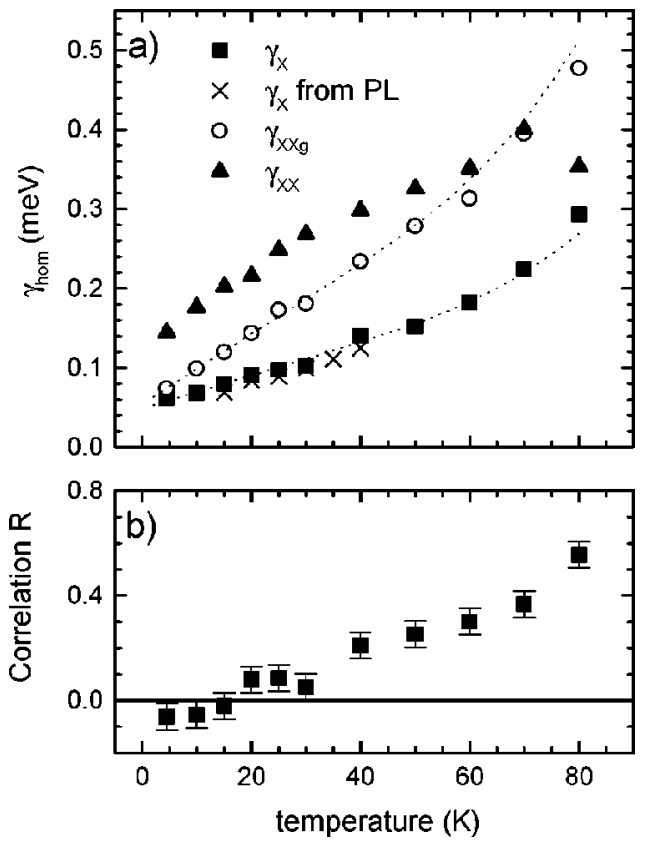

FIG. 3. (a) Temperature dependency of the linewidths of the exciton $\gamma_{X}$ (squares, crosses), biexciton $\gamma_{X X g}$ (circles), and exciton to biexciton $\gamma_{X X}$ (triangles) transition. The dotted lines are fits to $\gamma_{X}$ and $\gamma_{X X g}$ using Eq. (2). (b) Temperature dependency of the dephasing correlation $R$ calculated from Eq. (3).

width $\gamma_{0}$. For the optical phonon scattering, we used $b$ $=10 \mathrm{meV}$ and $E_{\mathrm{LO}}=36 \mathrm{meV}$ from literature, ${ }^{34}$ since the temperature range does not allow for an accurate determination of the LO-phonon coupling. $\gamma_{0}$ is found to be $\gamma_{0, X}=49$ $\pm 5 \mu \mathrm{eV}$. The radiative decay rate of excitons close to zone center in a 20-nm GaAs SQW with a cap layer thickness between QW and surface of multiples of $\lambda / 2$, which is applicable for the SQW investigated here, is calculated in Ref. 35 to be $\gamma_{\mathrm{rad}}=60 \mu \mathrm{eV}$. We thus assume that $\gamma_{0, X}$ is due to radiative decay and that other scattering processes are negligible in this limit. The acoustic-phonon coefficient is found to be $a_{X}=2.1 \pm 0.3 \mu \mathrm{eV} / \mathrm{K}$, comparable to values reported in literature. ${ }^{36,34}$

The same fit to the biexciton dephasing $\gamma_{X X g}$ with $b$ $=20 \mathrm{meV}$ yields $\gamma_{0, X X g}=55 \pm 5 \mu \mathrm{eV}$ and $a_{X X g}=4.4$ $\pm 0.5 \mu \mathrm{eV} / \mathrm{K}$. In view of the absence of additional scattering processes for the exciton, we assume that also for the biexciton $\gamma_{0, X X}$ represents its radiative broadening. The radiative decay of quasi-two-dimensional biexcitons in the giant oscillator strength model depends on the size of the biexciton $\Lambda_{X X} \cdot{ }^{18}$ In the limit of no biexciton binding, the biexciton is large compared to the light wavelength $\lambda$ in the material $(\approx 250 \mathrm{~nm})$, and the radiative decay rate is twice the one of the exciton. In the limit of a small biexciton $\left(2 \pi \Lambda_{X X} \ll \lambda\right)$, the radiative rate scales like $\Lambda_{X X}^{-2}$. The investigated sample is in the transition region $\left(2 \pi \Lambda_{X X} \approx \lambda\right)$, where a radiative rate close to the one of the exciton was calculated ${ }^{19}$ in agreement with our experimental finding. In contrast, the bipolariton model for the decay of quasi-two-dimensional biexcitons predicts a fast decay $\left(\gamma_{X X g} \approx 0.3 \mathrm{meV}\right)$ of the biexciton into interface polaritons, ${ }^{19}$ which is not in agreement with the observed biexciton linewidth.

The acoustic-phonon coefficient $a_{X X g}$ is about twice the one of the exciton. The deformation potential, that is the dominating interaction between excitons and acoustic phonons, is for biexcitons twice the one of excitons, since they consist of two electron-hole pairs. This would explain the observed ratio. However, the dispersions of exciton and biexciton are different due to their different masses, which is also expected to influence the scattering efficiency.

The relation between the linewidth of the excitonbiexciton transition $\gamma_{X X}$ and the line-widths of exciton $\gamma_{X}$ and biexciton $\gamma_{X X g}$ contains information about the correlation of the scattering events causing $\gamma_{X}$ and $\gamma_{X X g}$. If $\gamma_{X}$ and $\gamma_{X X g}$ are due to normal distributions of elastic Markovian scattering events with the correlation $R$, one finds ${ }^{37}$

$$
\gamma_{X X}=\gamma_{X}+\gamma_{X X g}-2 R \sqrt{\gamma_{X} \gamma_{X X g}},|R| \leqslant 1 .
$$

Here $R=0$ represents uncorrelated scattering, for which $\gamma_{X X}$ is the sum of $\gamma_{X}$ and $\gamma_{X X g}$. Using this relation, we have calculated $R$ from the experimental data [Fig. 3(b)]. For low temperatures, $\gamma_{X X g}$ and $\gamma_{X}$ are virtually uncorrelated. This is expected for the radiative decay ${ }^{38}$ since the photon modes that couple to the transitions are different in energy. With increasing temperature the correlation increases, which shows that phonon scattering of excitons and biexcitons are correlated. Reported data on $\gamma_{X}$ and $\gamma_{X X}$ in inhomogeneously broadened $\operatorname{In}_{x} \mathrm{Ga}_{1-x}$ As QW's (Ref. 11) are in agreement with this result. It allows the conclusion that the phonon modes involved in the scattering of exciton and biexciton are in a significant part equal. For phonon absorption processes, momentum and energy conservation selects different phonon modes for the different dispersions of exciton and biexciton. Energy conservation is slightly relaxed by the radiative broadening, which is comparable to the differences in the dispersions at the smallest interacting phonon energy of about $100 \mu \mathrm{eV}$ for the exciton and $200 \mu \mathrm{eV}$ for the biexciton. At the largest interacting phonon energy of about $1 \mathrm{meV}$ given by the width of the SQW, the dispersion energy differences are well above this broadening. We thus expect mainly different phonon modes to be absorbed from exciton and biexciton. For elastic phonon scattering, the selection rules are relaxed, so that a phonon mode can interact with both resonances. We thus take the correlation of the acoustic phonon scattering of excitons and biexcitons as a hint that elastic processes play an important role in the scattering. However, a more detailed microscopic description of the phonon dephasing is necessary.

In conclusion, the linewidths of quasi-two-dimensional excitons and biexcitons have been measured and attributed to radiative decay and phonon scattering. The determined radiative rates of exciton and biexciton are similar, and both are in agreement with recent calculations. ${ }^{35,19}$ A fast biexcitonic decay into interface polaritons predicted by Ivanov, Haug, and Keldysh ${ }^{21}$ is not observed. Using the linewidth of the exciton-biexciton transition, correlations between the scattering of exciton and biexciton are disclosed. It is found that the radiative decay of exciton and biexciton is uncorrelated, as expected due to the different involved photon energies. The phonon scattering of exciton and biexciton instead show a 
strong correlation, indicating the importance of elastic phonon scattering. These results are significant for many theoretical approaches describing the nonlinear optical response of semiconductors close to the band edge, in which approximations for the relative dephasing rates are used. ${ }^{39,32}$
The authors want to thank C. B. Sørensen (III-V Nanolab) for growing the high-quality GaAs sample and P. Borri for helpful discussions. III-V Nanolab is a joint laboratory between the Research Center COM and the Niels Bohr Institute, Copenhagen University.
${ }^{1}$ R. Miller, D. Kleinman, A. Gossard, and O. Munteanu, Phys. Rev. B 25, 6545 (1982).

${ }^{2}$ D. Birkedal, J. Singh, V. G. Lyssenko, J. Erland, and J. M. Hvam, Phys. Rev. Lett. 76, 672 (1996).

${ }^{3}$ W. Langbein and J. M. Hvam, Phys. Rev. B 59, 15405 (1999).

${ }^{4}$ D. S. Chemla, A. Maruani, and E. Batifol, Phys. Rev. Lett. 42, 1075 (1979).

${ }^{5}$ J. M. Hvam, I. Balslev, and B. Honerlage, Europhys. Lett. 4, 839 (1987).

${ }^{6}$ H. Akiyama, T. Kuga, M. Matsuoka, and M. Kuwata-Gonokami, Phys. Rev. B 42, 5621 (1990).

7 J. Shah, in Ultrafast Spectroscopy of Semiconductors and Semiconductor Nanostructures (Springer, Berlin, 1996), Chap. 2.

${ }^{8}$ S. T. Cundiff, Phys. Rev. A 49, 3114 (1994).

${ }^{9}$ W. Langbein, J. M. Hvam, M. Umlauff, H. Kalt, B. Jobst, and D. Hommel, Phys. Rev. B 55, R7383 (1997).

${ }^{10}$ H. Nickolaus and F. Henneberger, Phys. Rev. B 57, 8774 (1998).

11 P. Borri, W. Langbein, J. M. Hvam, and F. Martelli, Phys. Rev. B 60, 4505 (1999).

${ }^{12}$ M. Joschko, M. Wörner, T. Elsässer, E. Binder, T. Kuhn, R. Hey, H. Kostial, and K. Ploog, Phys. Rev. Lett. 78, 737 (1997).

${ }^{13}$ H. Wang, J. Shah, T. Damen, and L. Pfeiffer, Phys. Rev. Lett. 74, 3065 (1995).

${ }^{14}$ P. Leisching, T. Dekorsy, H. J. Bakker, H. G. Roskos, H. Kurz, and K. Köhler, J. Opt. Soc. Am. B 13, 1009 (1996).

${ }^{15}$ M. Hasuo, H. Kawano, and N. Nagasawa, J. Lumin. 66\&67, 396 (1996).

${ }^{16}$ A. L. Ivanov, M. Hasuo, N. Nagasawa, and H. Haug, Phys. Rev. B 52, 11017 (1995).

${ }^{17}$ C. Dörnfeld and J. M. Hvam, IEEE J. Quantum Electron. 25, 904 (1989).

${ }^{18}$ D. S. Citrin, Phys. Rev. B 50, 17655 (1994).

${ }^{19}$ A. L. Ivanov, H. Wang, J. Shah, T. C. Damen, L. V. Keldysh, H. Haug, and L. N. Pfeiffer, Phys. Rev. B 56, 3941 (1997).

${ }^{20}$ A. L. Ivanov and H. Haug, Phys. Rev. Lett. 74, 438 (1995).

${ }^{21}$ A. L. Ivanov, H. Haug, and L. V. Keldysh, Phys. Rep. 296, 237 (1998).

${ }^{22}$ K. Bott, O. Heller, D. Bennhardt, S. Cundiff, P. Thomas, E.
Mayer, G. Smith, R. Eccleston, J. Kuhl, and K. Ploog, Phys. Rev. B 48, 17418 (1993).

${ }^{23}$ A. Paul, J. Bolger, A. Smirl, and J. Pellegrino, J. Opt. Soc. Am. B 13, 1016 (1996).

${ }^{24}$ Y. Z. Hu, R. Binder, S. W. Koch, S. T. Cundiff, H. Wang, and D. G. Steel, Phys. Rev. B 49, 14382 (1994).

${ }^{25}$ H. Wang, K. B. Ferrio, D. G. Steel, P. R. Berman, Y. Z. Hu, R. Binder, and S. W. Koch, Phys. Rev. B 49, R1551 (1994).

${ }^{26}$ M. Wegener, D. Chemla, S. Schmitt-Rink, and W. Schäfer, Phys. Rev. A 42, 5675 (1990).

${ }^{27}$ K. Ferrio and D. Steel, Phys. Rev. B 54, R5231 (1996).

${ }^{28}$ W. Langbein, P. Borri, and J. Hvam, in Radiative Processes and Dephasing in Semiconductors, edited by D. Citrin (Optical Society of America, Washington, D.C., 1998), Vol. 18, Chap. Nonlinear Optics and Ultrafast Dynamics, p. 11.

${ }^{29}$ P. Kner, S. Bar-Ad, M. V. Marquezini, D. S. Chemla, and W. Schäfer, Phys. Rev. Lett. 78, 1319 (1997).

${ }^{30}$ G. Bartels, A. Stahl, V. M. Axt, B. Haase, U. Neukirch, and J. Gutowski, Phys. Rev. Lett. 81, 5880 (1998).

${ }^{31}$ W. Schäfer, D. Kim, J. Shah, T. Damen, J. Cunningham, K. Goossen, L. Pfeiffer, and K. Köhler, Phys. Rev. B 53, 16429 (1996).

${ }^{32}$ T. Östreich, K. Schönhammer, and L. J. Sham, Phys. Rev. B 58, 12920 (1998).

${ }^{33}$ S. Rudin, T. L. Reinecke, and B. Segall, Phys. Rev. B 42, 11218 (1990).

${ }^{34}$ D. Gammon, S. Rudin, T. L. Reinecke, D. S. Katzer, and C. S. Kyono, Phys. Rev. B 51, 16785 (1995).

${ }^{35}$ S. Haas, T. Stroucken, M. Hübner, J. Kuhl, B. Grote, A. Knorr, F. Jahnke, S. W. Koch, R. Hey, and K. Ploog, Phys. Rev. B 57, 14 860 (1998).

${ }^{36}$ P. Borri, W. Langbein, J. M. Hvam, and F. Martelli, Phys. Rev. B 59, 2215 (1999).

${ }^{37}$ K. B. Ferrio and D. G. Steel, Phys. Rev. Lett. 80, 786 (1998).

${ }^{38}$ N. Bloembergen, H. Lotem, and R. Lynch, Indian J. Pure Appl. Phys. 16, 151 (1978).

${ }^{39}$ V. M. Axt and A. Stahl, Z. Phys. B: Condens. Matter 93, 205 (1994). 